\title{
The Rising Trend in Seropositivity among the Diverse Population of Karachi-Possible Implication in SARS-Cov-2 Control
}

Faiza Rezwan ${ }^{1 *}$, Samreen Kulsoom Zaidi ${ }^{2}$, Asma Danish ${ }^{2,8}$, Shabnum Khawaja ${ }^{3}$, Mehjabeen Imam ${ }^{4}$, Jawad Hassan ${ }^{5}$, Samra Waheed ${ }^{5,6}$, Arshi Naz ${ }^{7}$, Munira Borhany ${ }^{6,8}$, Tahir Shamsi ${ }^{8}$

${ }^{1}$ Department of Microbiology

${ }^{2}$ Department of Paediatrics and Paediatric Infectious Diseases

${ }^{3}$ Department of Chemical Pathology

${ }^{4}$ Department of Transplant Immunology

${ }^{5}$ Department of Haematology

${ }^{6}$ Department of Blood Bank

${ }^{7}$ Department of Research

${ }^{8}$ Department of Clinical Haematology \& BMT

DOI: $\underline{10.36348 / \mathrm{sjpm} .2020 . \mathrm{v} 05 \mathrm{i} 12.005}$

| Received: 08.11.2020 | Accepted: 19.11.2020 | Published: 16.12 .2020

*Corresponding author: Dr. Faiza Rezwan

\section{Abstract}

Background: There is a sharp decline in new SARS-CoV-2 cases in Karachi from July onwards; most of them were asymptomatic. Seroconversion rates vary markedly in different countries. Previously, 36\% of seropositivity was reported in the adult population of Karachi in July this year. The current study was conducted to report if seroprevalence has increased and has a possible role in community immunity. Methods: This was a prospective cross-sectional study conducted in the first and second weeks of September 2020 at the National Institute of Blood disease and Bone Marrow Transplantation Hospital (NIBD), Karachi, Pakistan. Blood samples were collected from different segments of the population of Karachi workforce/community. An anti-SARS-CoV-2 test was performed using ECLIA from Roche Diagnostics International. Results: A total of 2404 subjects' blood samples were received from healthcare workers, the industrial workforce, and healthy blood donors. Seroprevalence in industrial workers was highest (70\%) as compared to healthcare workers (40\%). Overall seropositivity in males $(39 \%)$ was higher than females (16\%). Blood donors (all males) showed seropositivity of $37.8 \%$. The comorbid state was not significantly associated with seropositivity (p-value $>0.05)$. Total cases of antibody-positive were 1322 of 2100 (55\%). Conclusion: Our results confirmed the current seroprevalence of $55 \%$ in the adult population in Karachi. There was a sharp rise from $36 \%$ reported previously in July. This rise coincided with a sharp decline in new reported COVID 19 cases. We can conclude that the adult population from Karachi has shown an upsurge in seropositivity which will certainly have a beneficial role in SARS-CoV-2 control in the future.

Keywords: COVID-19 disease, Seroprevalence, Asymptomatic, Seroconversion, Electro-Chemiluminescence Immunoassay (ECLIA).

Copyright (C) 2020 The Author(s): This is an open-access article distributed under the terms of the Creative Commons Attribution 4.0 International License (CC BY-NC 4.0) which permits unrestricted use, distribution, and reproduction in any medium for non-commercial use provided the original author and source are credited.

\section{INTRODUCTION}

After the commencement of the pandemic, SARS CoV-2 infection has now become a global health crisis. Almost every country of the world is striving vigorously to control its rate of spread by adopting strategies like strict and smart lockdowns, containment of the vulnerable population, pharmaceutical prevention, and vaccine development but currently, no methodology has yet produced a favorable outcome [1, $2]$.
Numerous clinical trials for vaccine development are currently underway; few have even started Phase-III, after a successful phase-I and II trial [3]. While promising results have provided hope, it is still unclear as to how long the studies will take to establish safety and efficacy. In the meantime building up herd immunity which is defined as indirect protection conferred to susceptible individuals when an abundant proportion of immune individuals exist in a population, through natural spread is a possible theoretical option [4]. According to WHO, there are no reports of achieving herd immunity from any country and the world is still far from reaching the level of 
required immunity to curtail further spread of SARSCoV-2 infection [5].

Pakistan has seen a dramatic rise and fall of SARS CoV-2 infection rate since it was first reported by the Ministry of Health, Government of Pakistan on February 26, 2020, in Karachi, Sindh province imported by a man traveling from Iran [6]. This was followed by a more influx of people from across the borders with a subsequent increase in the local spread of infection. The government of Pakistan imposed a strict lockdown in response to the rising rates of infection by March 17th closing all areas of mass gatherings including mosques, schools, and shopping malls. These measures somewhat curbed the spread of infection in public places but local spread continued to increase owing to the dense population in low socioeconomic areas and social gatherings. Pakistan being a resource-limited country with a majority of its population being daily wagers, could not sustain the lockdown and by 23rd May after a review of the nation's declining financial situation "smart lockdown" i.e., highlighting and closing down of selective outbreaks, was enforced.

A surge of infections followed thereafter as people came out in massive numbers and failed to adhere to any standard operating procedure advised by the government. By mid-July, Pakistan had >250,000 confirmed cases and $>5000$ deaths with a fatality rate of $2 \%$ and it was expected to double by end of August. Our group has reported a seroprevalence of $36 \%$ in the adult population of Karachi in the month of July [7].

Dramatically, Pakistan saw a sharp decline in the number of positive cases along with deaths from the end of July to date, in spite of total relaxation of lockdown. This has led to various assumptions and theories justifying these numbers, herd immunity through infection being one of them. According to WHO only $10 \%$ of the global population has antibodies to SARS CoV-2 which is far away from achieving herd immunity [7]. Similar low seropositivity results have been reported from most of the developed world. Havers et al. have reported seroprevalence from the US ranging from $1.0 \%$ in San Francisco to $6.9 \%$ in New York City [8]. Likewise in the UK, the highest seroprevalence reported in London is $13 \%$ while in Belgium it's $4.2 \%[9,10]$. As, it's an already known fact that for herd immunity to attain, around $55-60 \%$ of the population needs to develop immunity against the virus, and no country has yet reported these numbers so far [4, 10].
In this background, we planned a follow-up seroprevalence study in the adult population of Karachi to determine the change in previous immune status after a period of two months while there was a sharp decline in new RT-PCR positive cases to COVID-19 desk at National Command and Operating Center (NCOC), Pakistan [5].

\section{MATERIAL AND METHODS}

This was a prospective cross-sectional study conducted in the first and second week of September 2020 at the National Institute of Blood disease and Bone Marrow Transplantation Hospital (NIBD) at Karachi, Pakistan, after approval from the institution's ethical review committee. Clinical information proforma was filled for each subject identifying chronic disorders, previous covid-19 infection, etc. Blood samples were collected from different groups of Karachi workforce/community after taking informed consent. The study included adult male and female participants from different segments of the population. RT-PCR proven COVID-19 cases were excluded. They have been categorized into three groups including Industrial workers, healthcare workers, and healthy voluntary blood donors. A Three-milliliter blood sample was collected in a vacutainer tube from all participants. The anti-SARS-CoV-2 test was performed by the Electro-Chemiluminescence immunoassay (ECLIA) method on the Cobas e-411Immunoassay analyzer by Roche diagnostics International Ltd by Rotkreuz Switzerland. It is a non-quantitative total antibody test (including $\operatorname{IgG}, \operatorname{IgM}$, and $\operatorname{Ig} \mathrm{A}$ ) for the detection of the presence of antibodies against the SARS-CoV-2 virus. This assay uses a double-antigen sandwich technique to detect antibodies against nucleocapsid (N) proteins of the COVID-19 virus. The manufacturer recommended to express the results as reactive if the Cut-of-Index (COI) value was $>1.0$ and Non-Reactive if COI was $<1.0$.

\section{STATISTICAL ANALYSIS}

Descriptive statistics were used to document frequencies, mean and median using SPSS package 23. A Chi-square test was used to compares two variables. A value of $<0.05$ was considered significant.

\section{RESULTS}

A total of 2404 samples were received including 478 health care workers, 505blood donors, and 1118 industrial employees as shown in Table 1. A total of 303 samples were also collected from haemodialysis patients. There were 1720 males and 684 females. Mean age was 35.27 years +/ 13.7 SD. 


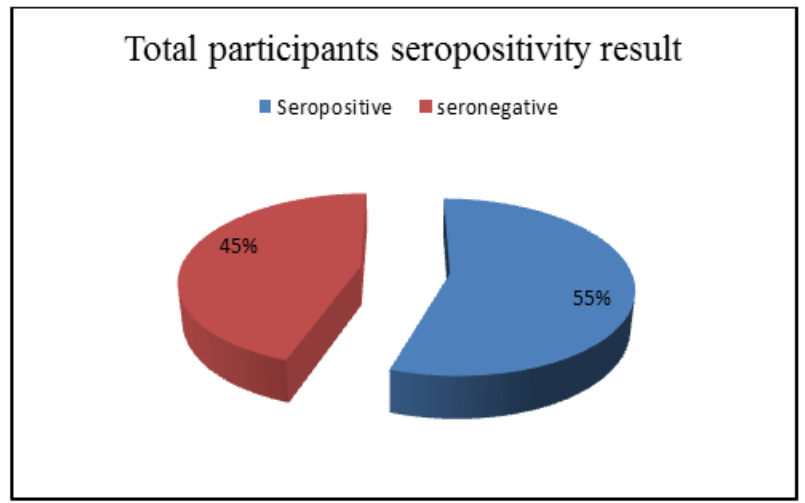

Fig-1: The above figure is showing the seropositivity result for SARS-CoV-2 antibodies in adult population of Karachi

The most remarkable finding to emerge from the data was that out of 2404 subjects, 1322 participants were tested positive which was around $55 \%$ as presented in figure 1 . Out of 1322 seropositive cases, $937(54.4 \%)$ male and $384(56.1 \%)$ female subjects were positive.

\section{Seroprevalence in adult population of Karachi}

\begin{tabular}{|l|l|l|l|}
\hline \multicolumn{1}{|c|}{ Study subjects } & \multicolumn{3}{|c|}{ SARS-CoV-2 Antibodies Result } \\
\hline & Negative & Positive & Total \\
\hline Blood donor & 314 & 191 & 505 \\
\hline Dialysis patients & 184 & 118 & 303 \\
\hline HCW & 242 & 234 & 478 \\
\hline Industrial Worker & 339 & 779 & 1118 \\
\hline Total & 1079 & 1322 & 2404 \\
\hline
\end{tabular}

Table-1: The above table shows the total number of subjects included in study along with their seropositivity result for Anti SARS-CoV-2 antibodies

Table 1 presents the breakdown of participants according to antibodies positive results. As shown in table 1 , one hundred ninety-one blood donors were confirmed positive for antibodies that make it around $37.8 \%$. While referring to $\mathrm{HCW}$ findings, the sero positivity was detected in 234 subjects that were $50 \%$ approximately as explained in table 1 . The most noteworthy finding was observed in industrial employees formulating approximately $70 \%$ of sero positivity with 779 persons reported positive. Among the patient population visiting a dialysis centre in Karachi for haemodialysis, nearly $40 \%$ of sero prevalence against SARS-COV-2 was detected. There is so statistical significance among Co-morbid states and seropositivity. (P-value $>0.05$ ) The correlation between Blood donors, HCWs and industrial employees results were verified using chi-square test. The chisquare test showed significant differences between blood donors, dialysis patients and industrial workers results. P-value of $<0.05$ is considered significant. The differences between seropositive and seronegative population in percentage is also highlighted in Figure 1.Theobservation to emerge from the data comparison in Figure 1 was the highest seropositivity in industrial employees followed by healthcare workers.

\section{Seropositivity in various population of Karachi}

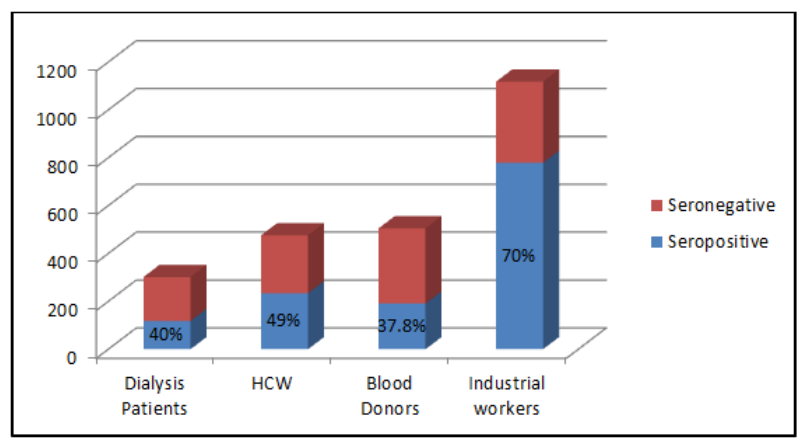

Fig-2: The above graph is illustrating the percentage seropositivity among dialysis patients, blood donors, healthcare workers and industrial employees

Overall, these results indicated that the seroprevalence has progressively risen and there was an upsurge in seroprevalence among healthcare workers, industrial employees and community person in September which might have implications in SARSCoV-2 control.

\section{DISCUSSION}

This study was conducted to check the difference of seropositivity among different adult population strata in Karachi as compared to what was reported in July 2020 [7]. As compared to previously reported 36 percent, there was a sharp rise in SARSCoV-2 seropositivity to $55 \%$ in the population groups assessed in September 2020. The worldwide different serology based studies have shown wide variations in seroprevalence i.e., from $1 \%$ to $9 \%$ [11-14]. Herd immunity is considered as a target that is achieved when the majority (60-70\%) of the population develops immunity against a given infectious agent, resulting in limitation in further spread of the disease. Prior studies have noted the importance of herd immunity and its impact on the epidemiology of infectious diseases and their prevention and control by vaccine [15]. The Herd immunity concept was discovered in 1930s while observing the cohort of children developing immunity to measles [16]. Similarly, herd immunity has also been mentioned in the literature against rubella, chicken pox, mumps, polio and pertussis and predicting their long term impact of vaccination program [17]. Therefore, considering these historical perspectives of infectious diseases, there is a probability that worldwide herd immunity against SARS-CoV-2 can also be achieved by vaccination or through widespread asymptomatic / mildly symptomatic infection.

The most obvious and relevant finding to emerge from the analysis was that our study population have observed sharp rise in seroprevalence from $36 \%$ to $55 \%$ from July to September. This finding signifies that the seroprevalence is progressively rising and which foresees a possibility of achieving herd immunity in Karachi in near future. Although it is still unknown 
whether these antibodies will last long enough period to provide protection in subsequent re-exposure to same or a mutant strain of COVID-19 virus infection. If we closely assess the seropositivity rate in different working strata then we found that marked increase has been detected in HCW from $13 \%$ in July to $40 \%$ in month of September.

Earlier unpublished data by Chughtai et al. also reported the $32 \%$ of seropositivity in HCW which is consistent with our current findings [18].This result may also be explained by the fact that frontline healthcare workers were included in our current study in HCW group. Besides, the noticeable observation was the persistent rise in seroconversion in industrial workers mounting from $50 \%$ to $70 \%$. The reason for this is not very clear, however, the assumption behind this persistent rise could be the relaxation in lockdown, regular working hours and lack of social distancing and preventive measures because of their poor living standards in slum areas where most of them live. Another important finding is in our third group which encompasses blood donors and high risk community population i.e., haemodialysis patients who are more prone to complications after SARS-CoV-2 infection. This segment of population showed seroprevalence percentage of around $40 \%$. Further investigation revealed that the majority of haemodialysis patients were either asymptomatic or had mild manifestations of infection. In addition, blood donors which represented healthy people of community have $38 \%$ of seropositivity.

In reviewing the literature, Nisar et al. has also reported similar rise in seropositivity in community population from $0.2 \%$ and $0.4 \%$ in April to $8.7 \%$ and $15.1 \%$ in low-and high-transmission-areas respectively [19]. Demographic data of Pakistan indicates that the median age of population is 22 which could be a key factor in preventing severe and critical disease and increased seropositivity due to mild or asymptomatic infections and our study age range findings is also validating this assumption [20].

One important question is how such a large population seroconverted without producing symptoms? There are several other possible explanations for this finding. One possible reason is the practice of BCG immunization in our population because of high endemicity of tuberculosis as part of immunisation programme since last four decades [21]. Studies have pointed out the importance of BCG in protecting the population against severe SARS-CoV-2 infection [22]. This also accords with some earlier observations, which showed that there is decrease in various viral infections due to Bacillus CalmetteGuérin (BCG) vaccination an effect might be due to trained immunity [23].Therefore, it can be hypothesized that BCG vaccine might lead to increased number of mild or asymptomatic cases resulting in high seropositivity.

Another possible explanation for this largescale seroconversion is vaccination against measles may have a protective effect. Similarity between amino acid sequence of spike protein of SARS-CoV-2 and fusion protein of measles virus as stated in one of the studies might have offered cross-protection [24, 25]. Overall decreases in incidence of other viral illnesses have been reported after MMR vaccine; due to the strengthening of body immune system in general [25]. Prior helminth infections have also been associated with reduced risk of severe COVID 19 [26].

In addition, mutations in any virus are the key factors in defining the variability in disease transmission, progression and severity. Also literature review reveals that SARS-CoV-2, has developed various mutations resulting in creation of different variants or clades [27]. Ganchi et al. has also reported the presence of mutations in SARS-CoV-2 strains of our country which signifies their diversity in our population [28]. These mutations can also have a significant role in disease severity as observed in certain developed countries with the S D614G mutation in SARS-CoV-2 strains leading to high case fatalities [29]. It can thus be suggested that mutated strains in our country might cause the mild or asymptomatic cases which led to high seropositivity.

As WHO is emphasizing of difficulty in acquiring herd immunity in the world through natural infection but in our population setting the seropositivity percentage of $55 \%$ is describing alternate picture [30]. Since herd immunity threshold depends upon reproductive number of virus i.e., $R_{o}$ and it also varies with population density and other transmission dynamics. ${ }^{32}$ In current pandemic, it is assumed that $R_{o}$ of 2-3 will need herd immunity threshold of 60-70\%.On the other hand, WHO and some studies recently stated that herd immunity with SARS-COV-2 could be achieved with $50 \%$ [31]. Thus in our study, findings $55 \%$ of seroprevalence in Karachi is giving us hope of attaining herd immunity. However, these data must be interpreted with caution because it is a small scale study with limitation in our sample population.

In general, it seems that SARS-CoV-2 infection has reached to endemicity in our population; a widespread infection affecting a large population segment without any severity and complications. Affected individuals remain mainly asymptomatic or develop milder symptoms. This endemicity will continue to affect our population; either leading to herd immunity provided the antibodies protective efficacy is long lasting. Currently the extent to which population will develop neutralizing immunity against SARSCoV-2 is unclear. 
Since SARS-CoV-2 is a new pathogen hence its salient features regarding transmission and infectivity is still unknown. Thus, our above study provides an assumption that we might attain herd immunity via natural infection. However a large scale study is warranted to validate our assumption. Now even if we consider that our large number of population is immune then the recommendation regarding vaccination will arise in future. And vaccine is the safe way of providing effective herd immunity. As observed in literature review, severe infections are associated more with elderly population and patients with co morbidities and immunocompromised conditions [32]. So, an importance should be placed on policies that protect the most susceptible individuals. Therefore, those persons with co-morbidities and compromised immune system will remain potentially at risk to develop severe or life-threatening disease and would surely need a vaccine. Furthermore seronegative individuals willing to travel abroad, and seronegative children, adolescents, frontline healthcare workers and young adults may also benefit from vaccine. Despite these promising results additional work is required to establish the protective efficacy of antibodies and also further research should be undertaken on a nationwide level to investigate the seroprevalence against SARSCoV-2 in our country.

\section{Acknowledgement:}

We would like to thank Mr. Shakir Ahmed for his contribution in data compilation.

\section{REFERENCES}

1. Bock, W., Adamik, B., Bawiec, M., Bezborodov, V., Bodych, M., Burgard, J. P., ... \& Ozanski, T. (2020). Mitigation and herd immunity strategy for COVID-19 is likely to fail. medRxiv.

2. Flaxman, S., Mishra, S., Gandy, A., Unwin, H. J. T., Coupland, H., Mellan, T. A., ... \& Schmit, N. (2020). Estimating the number of infections and the impact of non-pharmaceutical interventions on COVID-19 in European countries: technical description update. arXiv preprint arXiv:2004.11342.

3. Folegatti, P. M., Ewer, K. J., Aley, P. K., Angus, B., Becker, S., Belij-Rammerstorfer, S., ... \& Dold, C. (2020). Safety and immunogenicity of the ChAdOx1 nCoV-19 vaccine against SARS-CoV-2: a preliminary report of a phase $1 / 2$, single-blind, randomised controlled trial. The Lancet, 396(10249), 467-478.

4. Randolph, H. E., \& Barreiro, L. B. (2020). Herd Immunity: Understanding COVID19. Immunity, 52(5), 737-741.

5. WHO, Coronavirus disease (COVID-19) Weekly Epidemiological Update and Weekly Operational Update. Available from:https://www.who.int/emergencies/diseases/no vel-coronavirus-2019/situation-reports $(20$
6. Government of Pakistan. Available from: http://covid.gov.pk (18 September 2020, date last accessed).

7. Zaidi, S., Rizwan, F., Riaz, Q., Siddiqui, A., Khawaja, S., Imam, M., ... \& Shamsi, T. (2020). Seroprevalence of anti-SARS-CoV-2 antibodies in residents of Karachi-challenges in acquiring herd immunity for COVID 19. Journal of Public Health.

8. Havers, F. P., Reed, C., Lim, T. W., Montgomery, J. M., Klena, J. D., Hall, A. J., ... \& Krapiunaya, I. (2020). Seroprevalence of antibodies to SARSCoV-2 in six sites in the United States, March 23May 3, 2020. medRxiv.

9. Ward, H., Atchison, C. J., Whitaker, M., Ainslie, K. E., Elliot, J., Okell, L. C., ... \& Darzi, A. (2020). Antibody prevalence for SARS-CoV-2 in England following first peak of the pandemic: REACT2 study in 100,000 adults. MedRxiv.

10. Herzog, S., De Bie, J., Abrams, S., Wouters, I., Ekinci, E., Patteet, L., ... \& Hens, N. (2020). Seroprevalence of $\operatorname{IgG}$ antibodies against SARS coronavirus 2 in Belgium: a prospective crosssectional study of residual samples. medRxiv.

11. Pollán, M., Pérez-Gómez, B., Pastor-Barriuso, R., Oteo, J., Hernán, M. A., Pérez-Olmeda, M., ... \& Molina, M. (2020). Prevalence of SARS-CoV-2 in Spain (ENE-COVID): a nationwide, populationbased seroepidemiological study. The Lancet, 396(10250), 535-544.

12. Shakiba, M., Nazari, S. S. H., Mehrabian, F., Rezvani, S. M., Ghasempour, Z., \& Heidarzadeh, A. (2020). Seroprevalence of COVID-19 virus infection in Guilan province, Iran. medRxiv.

13. Biggs, H. M., Harris, J. B., Breakwell, L., Dahlgren, F. S., Abedi, G. R., Szablewski, C. M., ... \& Gilani, Z. (2020). Estimated community seroprevalence of SARS-CoV-2 antibodies - two Georgia counties, April 28-May 3, 2020. Morbidity and Mortality Weekly Report, 69(29), 965.

14. Stringhini, S., Wisniak, A., Piumatti, G., Azman, A. S., Lauer, S. A., Baysson, H., ... \& Arm-Vernez, I. (2020). Repeated seroprevalence of anti-SARSCoV-2 IgG antibodies in a population-based sample from Geneva, Switzerland. medRxiv.

15. Fine, P., Eames, K., \& Heymann, D. L. (2011). "Herd immunity": a rough guide. Clinical infectious diseases, 52(7), 911-916.

16. Hedrich, A. W. (1933). MONTHLY ESTIMATES OF THE CHILD POPULATION "SUSCEPTIBLE'TO MEASLES, 1900-1931, BALTIMORE, MD. American Journal of Epidemiology, 17(3), 613-636.

17. Anderson, R. M., \& May, R. M. (1985). Vaccination and herd immunity to infectious diseases. Nature, 318(6044), 323-329.

18. Chughtai, O. (2020). Preliminary results of the at Chughtai Lab survey of doctors / nurses / allied healthcare workers across Pakistan for COVID antibodies. [Internet]. 2020 [cited 5August 2020]: 
https://twitter.

com/OmarChughtai/status/1290939416840736768

(5 August 2020, date last accessed).

19. Nisar, M. I., Ansari, N., Amin, M., Khalid, F., Hotwani, A., Rehman, N., ... \& Iqbal, J. (2020). Serial household serosurvey for COVID-19 in low and high transmission neighborhoods of urban Pakistan. medRxiv.

20. Pakistan Demographics. (2020). (Population, Age, Sex, Trends) - Worldometer [Internet]. Worldometers.info. 2020 [cited 25 September 2020]. Available from: https://www.worldometers.info/demographics/paki stan-demographics/

21. Hasan, Z., Irfan, M., Khan, J. A., Jahangir, S. K., Haris, M., Ashraf, M., ... \& Rao, N. A. (2012). BCG vaccination is associated with decreased severity of tuberculosis in Pakistan. International journal of mycobacteriology, 1(4), 201-206.

22. Escobar, L. E., Molina-Cruz, A., \& Barillas-Mury, C. (2020). BCG vaccine protection from severe coronavirus disease 2019 (COVID19). Proceedings of the National Academy of Sciences, 117(30), 17720-17726.

23. O’Neill, L. A., \& Netea, M. G. (2020). BCGinduced trained immunity: can it offer protection against COVID-19?. Nature Reviews Immunology, 20(6), 335-337.

24. Sidiq, K. R., Sabir, D. K., Ali, S. M., \& Kodzius, R. (2020). Does Early Childhood Vaccination Protect Against COVID-19?. Frontiers in Molecular Biosciences, 7, 120.

25. Atabani, S. F., Obeid, O. E., Chargelegue, D., Aaby, P., Whittle, H., \& Steward, M. W. (1997). Identification of an immunodominant neutralizing and protective epitope from measles virus fusion protein by using human sera from acute infection. Journal of virology, 71(10), 7240-7245.

26. Hays, R., Pierce, D., Giacomin, P., Loukas, A., Bourke, P., \& McDermott, R. (2020). Helminth coinfection and COVID-19: An alternate hypothesis. PLoS Neglected Tropical Diseases, 14(8), e0008628.

27. Yang, X., Dong, N., Chan, E. W. C., \& Chen, S. (2020). Genetic cluster analysis of SARS-CoV-2 and the identification of those responsible for the major outbreaks in various countries. Emerging Microbes \& Infections, 9(1), 1287-1299.

28. Ghanchi, N. K., Masood, K. I., Nasir, A., Khan, W., Abidi, S. H., Shahid, S., ... \& Islam, N. (2020). SARS-CoV-2 genome analysis of strains in Pakistan reveals $\mathrm{GH}, \mathrm{S}$ and L clade strains at the start of the pandemic. bioRxiv.

29. Eaaswarkhanth, M., Al Madhoun, A., \& Al-Mulla, F. (2020). Could the D614 G substitution in the SARS-CoV-2 spike (S) protein be associated with higher COVID-19 mortality?. International Journal of Infectious Diseases.

30. Arora, R. K., Joseph, A., Van Wyk, J., Rocco, S., Atmaja, A., May, E., ... \& Williamson, T. (2020). SeroTracker: a global SARS-CoV-2 seroprevalence dashboard. The Lancet. Infectious Diseases.

31. Fontanet, A., \& Cauchemez, S. (2020). COVID-19 herd immunity: where are we? Nature Reviews Immunology, 20(10), 583-584.

32. Zhou, F., Yu, T., Du, R., Fan, G., Liu, Y., Liu, Z., \& Guan, L. (2020). Clinical course and risk factors for mortality of adult inpatients with COVID-19 in Wuhan, China: a retrospective cohort study. The lancet. 